\title{
INTERACCIONES ENTRE LA TELEVISIÓN Y SU AUDIENCIA SOCIAL: HACIA UNA CONCEPTUALIZACIÓN COMUNICACIONAL
}

\author{
Interactions between television and its \\ social audience: Towards a communication \\ conceptualization
}

Daniel Halpern, Natalia Quintas-Froufe y Francisco Fernández-Medina
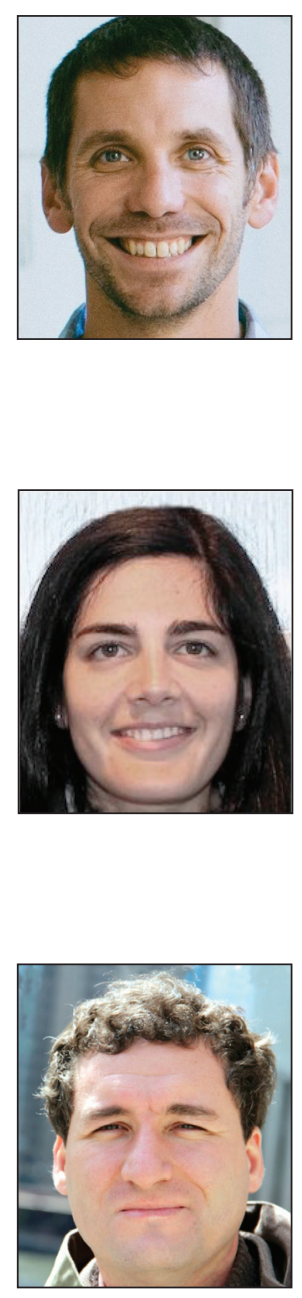

\section{Resumen}

Daniel Halpern es PhD en information and communication sciences. Profesor de la Facultad de Comunicaciones de la Pontificia Universidad Católica de Chile y director del think tank TrenDigital. Sus artículos han sido publicados en prestigiosas revistas académicas como Computers in human behavior y Behaviour and information technology. Su investigación se centra en las consecuencias sociales del uso de las tecnologías de información y comunicación. Ha asesorado a numerosas organizaciones y empresas en la implementación de redes sociales y desarrollo de nuevas aplicaciones.

http://orcid.org/0000-0002-1569-9876

Pontificia Universidad Católica de Chile, Facultad de Comunicaciones Alameda, 340. Santiago, Chile dmhalper@uc.cl

Natalia Quintas-Froufe es licenciada en publicidad y relaciones públicas por la Universidad de Vigo y doctora por la misma universidad. Profesora de estudio de las audiencias en la Facultad de Comunicación de la Universidade da Coruña desde 2010. Su investigación gira en torno a las transformaciones de la audiencia televisiva en el actual ecosistema mediático, fruto de la cual ha publicado numerosos artículos en varias publicaciones nacionales e internacionales.

http://orcid.org/0000-0001-7597-6516

Universidade da Coruña, Facultad de Comunicación Campus de Elviña, 15007 A Coruña, España n.quintas.froufe@udc.es

\begin{abstract}
Francisco Fernández-Medina es doctor en ciencias de la información y Master en comunicación y educación por la Universitat Autònoma de Barcelona. Periodista por la Pontificia Universidad Católica de Chile, trabaja como académico en el área de contenidos digitales interactivos en la Facultad de Comunicaciones de la misma universidad, donde dirige el diplomado en desarrollo y gestión de contenidos digitales. Tiene investigaciones en televisión digital interactiva, ha participado en proyectos de I+D y publicado en temas de economía política de la comunicación, industria audiovisual y plataformas digitales de distribución de contenidos.
\end{abstract}

http://orcid.org/0000-0001-6146-0375

Pontificia Universidad Católica de Chile, Facultad de Comunicaciones Alameda, 340. Santiago, Chile. ffernandez@uc.cl

Esta investigación estudia las interacciones entre la televisión y las redes sociales (lo que podríamos llamar televisión social) en el entorno latinoamericano, con dos grandes propósitos. El primero es explorar los factores que explican cómo las redes sociales inciden en el consumo televisivo, y en segundo lugar cuantificar estos factores para tener un alcance más objetivo del fenómeno. Para ello se empleó un diseño de investigación mixto que combinó grupos de discusión y una encuesta representativa que arrojó cinco resultados: 1) Cuantos más comentarios leen los usuarios en redes sociales mayor es el efecto que tienen en su conducta televisiva; 2) La televisión abierta se comenta más que el cable, bajo demanda y webcasting; 3 ) Facebook y Twitter son las plataformas sociales que más se utilizan para comentar; 4) El humor y los aspectos informativos 
son las temáticas más comentadas; y 5) Mientras Facebook se utiliza para conocer lo que los más cercanos ven, Twitter para informarse sobre lo que el público opina.

\title{
Palabras clave
}

Televisión social; TV; Audiencias; Mecanismos de participación; Redes sociales; Dispositivos móviles; Sociedad multipantaIla; Telespectadores; Convergencia digital; Grupos de discusión; Encuestas.

\begin{abstract}
This paper aims to study the interactions between television and social networks (what we might call social TV) in Latin America, with two goals in mind: 1) Identifying how social media is affecting television consumption, including influential factors; and 2) Quantifying these factors in order to have a clear picture of the phenomenon. For this purpose we used a mixed methods approach, combining a focus group and national survey. The study yielded five major findings: 1) The more comments users read in social media the higher the effect on their television behavior; 2) Open television is discussed more than cable, on demand, and webcasting; 3) The most discussed topics are humor and informational subjects; 4) Facebook and Twitter are the most commonly used platforms to comment about television; 5) While Facebook is the most used site to learn about what friends are viewing on television, Twitter is the most used site to learn about what the general public thinks about television content.
\end{abstract}

\section{Keywords}

Social TV; Television; Audiences; Participation mechanisms; Social network sites (SNS); Mobile devices; Multiscreen society; Viewers; Digital convergence; Focus groups; Surveys.

Halpern, Daniel; Quintas-Froufe, Natalia; Fernández-Medina, Francisco (2016). "Interacciones entre la televisión y su audiencia social: hacia una conceptualización comunicacional". El profesional de la información, v. 25, n. 3, pp. 367-375.

http://dx.doi.org/10.3145/epi.2016.may.06

\section{Introducción}

La incorporación de las redes sociales a la experiencia televisiva ha dado lugar a una nueva concepción de la audiencia activa denominada audiencia social, que ha surgido como respuesta a la fragmentación del actual ecosistema mediático caracterizado por la cultura participativa y la convergencia digital (Quintas-Froufe; González-Neira, 2014).

La interacción de las audiencias con los medios de comunicación tradicionales (Webster; Ksiazek, 2012) se ha acentuado con la multiplicación de pantallas. Esto ha derivado en una experiencia multipantalla (Giglietto; Selva, 2014), donde el usuario/espectador lleva a cabo varias actividades (media multitasking), muchas veces vinculadas con la programación televisiva. El nuevo hábito mediático se ha propagado rápidamente, generando audiencias conectadas que comparten un espacio virtual en el que interactúan entre ellas en tiempo real. Por ello, la mayoría de canales y programas de televisión han creado sus propias segundas pantallas, en muchos casos en forma de aplicaciones para smartphones y tabletas (Giglietto; Selva, 2014).

El carácter social inherente al medio televisivo se ha potenciado con la audiencia activa, en la que los televidentes participan estableciendo una comunicación vertical con el programa/cadena, y horizontal con los demás usuarios. De ahí que la sociabilidad sea una de las principales particularidades de la televisión social, al buscar que los televidentes compartan con sus contactos y alcancen de esta forma una experiencia común (Shin, 2013). Así como antes las familias solían reunirse a ver la televisión en un espacio y tiempo común, la nueva televisión social está creando espacios virtuales que aumentan la interactividad en programaciones compartidas tanto cuando se transmiten en vivo como en diferido.
Las posibilidades de participación de los nuevos medios, como aseguran Masip et al. (2015), facilitan la producción de contenidos generados por los ciudadanos. En este escenario el espectador social adquiere otro rol como prosumer, al generar contenidos con el fin de compartirlos a través de medios sociales como Facebook o Twitter (García-Galera; Valdivia, 2014). Uno de los aspectos más interesantes reside en identificar los factores que inciden en el comportamiento de la audiencia social, como sus motivaciones y razones para comentar y leer comentarios de otros (Segado; Grandío; Fernández-Gómez, 2015).

\section{Así como antes las familias se reunían a ver la televisión en un espacio y tiempo común, la nueva televisión social crea es- pacios virtuales que aumentan la interac- tividad en programaciones compartidas}

Esta investigación tiene dos propósitos:

- explorar los factores y variables que explican la incidencia de los medios sociales en el consumo televisivo;

- cuantificar estos aspectos para tener un alcance más objetivo del fenómeno en el entorno latinoamericano, región en la que la penetración de internet aumentó un 142\% entre 2006 y 2014 (Cepal, 2015), y que cuenta además con un porcentaje significativamente mayor de usuarios que participan en redes sociales $(78,4 \%)$, inclusive más que en América del Norte (64,6\%) o Europa Occidental $(54,5 \%)$, a pesar de estar insertado en una sociedad con menores ingresos per capita (Cepal, 2015). 
La elección de Chile a su vez se debe a que posee el mayor número de usuarios en redes sociales por habitante de América Latina y tiene el mayor tiempo de consumo televisivo de la región: el tiempo de visionado de televisión abierta es de tres horas diarias (CNTV, 2014). Además, el equipamiento tecnológico de los hogares chilenos es cada vez mayor: cada hogar dispone de una media de 2,2 teléfonos móviles (el 71,8\% de estos dispositivos dispone de conexión a internet) y el número medio de televisores por hogar es de 2,6, teniendo el 69,3\% de esos televisores conexión directa a internet (CNTV, 2014).

\section{Marco teórico y estado de la cuestión}

El rápido crecimiento de la televisión social supuso el planteamiento de investigaciones procedentes de varias áreas de conocimiento. En el área de ciencias de la comunicación, y más concretamente en el análisis de audiencias, las aproximaciones al concepto de audiencia social se han centrado en casos vinculados a una zona geográfica determinada o a un programa televisivo en concreto (Segado; Grandío; Fernández-Gómez, 2015). Por ejemplo, en el entorno europeo:

- D’Heer y Verdegem (2014) observaron el programa de actualidad belga De Zevende Dag en Twitter;

- Larsson (2013) estudió cómo el espectador interactuó en Twitter en el talk show Hübinette de la televisión pública sueca;

- Highfield, Harrington y Bruns (2013) se centraron en Eurovisión y el uso de Twitter como canal de expresión transcontinental del fenómeno fan eurovisivo.

En España, donde la empresa Kantar Media lanzó una de las primeras mediciones oficiales de la audiencia social en Europa (Kantar Twitter TV Ratings), se han llevado a cabo diversos tipos de análisis.

Por una parte hay investigaciones que estudian cómo el usuario interactúa a través de las redes sociales en espacios televisivos como las series de ficción (Deltell-Escolar; Claes; Osteso-López, 2013; Gómez; Vidales, 2015), galas televisivas con gran impacto en Twitter (Congosto et al., 2013) o el falso documental (Quintas-Froufe; González-Neira; DíazGonzález, 2015; Ferreras-Rodríguez, 2014).

Por otra parte se han elaborado estudios para establecer el tipo y grado de correspondencia existente entre la audiencia social y la tradicional (Deltell-Escolar, 2014; GonzálezNeira; Quintas-Froufe, 2014) e investigaciones centradas en valorar las estrategias de éxito que se han puesto en práctica para aumentar la audiencia social (Saavedra; Rodríguez; Barón, 2015).

Por último, diversos autores han tratado de exponer y valorar otras métricas y parámetros seleccionados para la medición de la audiencia social (Gallego, 2013); y la utilización de las redes sociales como mecanismos aplicados al análisis de audiencias (Echegaray-Eizaguirre; Peñafiel-Saiz, 2013).

En Latinoamérica también se ha estudiado la irrupción de las redes sociales en el actual panorama televisivo desde el punto de vista del usuario (la interactividad de la audiencia con las pantallas) y cómo el escenario de convergencia mediática influye en las audiencias y los medios, pero sólo considerando la opinión de expertos (Orozco, 2013).

En Chile en cambio, las aproximaciones que se han realizado son limitadas con respecto a la audiencia social. Fábrega y Vega (2013) estudiaron el vínculo entre la medición tradicional de la audiencia televisiva con la actividad en Twitter, y la interrelación de los ratings de audiencia del programa solidario Teletón y la actividad de los usuarios en Twitter, concluyendo que cada punto del rating televisivo del programa se podía asociar a un incremento de 1,5 tuits por minuto para el conjunto de la transmisión y de 6 tuits por minuto en horario prime time. Más recientemente Miranda-Bustamante y Fernández-Medina (2015) examinaron el discurso de la audiencia social de Twitter en programas de entrevistas con carácter político emitidas en la televisión abierta chilena, y encontraron que la correspondencia entre los tuits era baja y más que conversar entre las partes, los usuarios se limitaban a opinar sin hacer referencia a tuits anteriores.

\section{Diseño de la investigación}

Se empleó un diseño de investigación mixto que combinó métodos inductivos-cualitativos con cuantitativos. Se planteó que la articulación de ambas técnicas era la perspectiva metodológica más adecuada para aproximarse al objeto de estudio ya que hasta el momento la mayoría de aportaciones publicadas sobre televisión y audiencia social son de carácter cualitativo o cuantitativo, pero no hay ninguna que combine ambos métodos. El estudio se diseñó en dos fases consecutivas durante el curso académico 2014-2015.

\section{Método del grupo de discusión}

La primera parte utilizó grupos de discusión con doce adultos jóvenes entre 18 y 24 años. La selección de participantes en el focus group se realizó entre estudiantes de la Facultad de Ciencias de las Comunicaciones de la Pontificia Universidad Católica de Chile buscando una representación equitativa entre hombres y mujeres. La decisión de la selección de este grupo de edad estuvo determinada por dos factores:

- es el grupo con el hábito más extendido de utilización de segunda pantalla en el visionado televisivo según la Encuesta Nacional de Televisión de Chile (CNTV, 2014);

- es el grupo que en mayor medida ha asimilado las nuevas formas de consumo audiovisual, usando para ello las tecnologías móviles y digitales (CNTV, 2014).

Por ello se decidió que este era el grupo de edad apropiado para ahondar en el conocimiento de esta audiencia, al ser el que ha recibido un mayor impacto de la televisión social. La finalidad de la fase inicial fue identificar el tipo de red social y de contenido que incidía en el comportamiento de la audiencia social. Se consideró que la técnica cualitativa más adecuada era el focus group con el fin de generar información sobre las experiencias y creencias de los participantes como parte de audiencia social tratando de averiguar sus hábitos, percepciones y motivaciones para interactuar (Morgan, 1996).

En los grupos de discusión se pidió a los participantes reflexionar sobre varios aspectos relacionados con la televisión social y su rol como espectadores sociales. Además del 
moderador estuvo presente otro miembro del equipo con el fin de realizar una observación directa de carácter no participante. Los bloques de preguntas se agruparon en torno a cuatro áreas temáticas asociadas a las principales variables:

1) Uso y complementariedad de las pantallas. Los investigadores en televisión social, como afirman Segado, Grandío y Fernández-Gómez (2015), han centrado su atención en los medios sociales como una segunda pantalla donde la experiencia del consumo televisivo se expande. Sin embargo, el vínculo entre pantallas no está del todo definido. Recientemente algunos investigadores se han interesado por determinar la interactividad que se produce entre las pantallas por parte de la audiencia social, sobre todo por el impacto que tienen en las campañas publicitarias insertadas en este medio (Pynta et al., 2014).

2) Principales contenidos, géneros y programas de la televisión social. El tema de los contenidos televisivos es un factor determinante confirmado en investigaciones anteriores (Arrojo-Baliña, 2013) y en el propio grupo de discusión, donde los participantes manifestaron que su participación en la conversación social estaba fuertemente condicionada por el género y tema del programa.

Los espectadores comentan con mayor frecuencia en programación de televisión abierta que en la de pago

3) Hábitos televisivos y patrones de conducta de la audiencia social. La finalidad de este bloque de preguntas era identificar pautas de conducta del consumo televisivo social (horario, frecuencia, tiempo y soporte de conexión, etc.) y valorar cómo el componente social del consumo influye en los hábitos de la audiencia. Se trataba de determinar cómo el interés por lo que ven los amigos y familiares y las recomendaciones que éstos realizan intervienen en el consumo televisivo tradicional.

4) Rol de las redes sociales. La finalidad era identificar cuáles son las redes sociales que más se utilizaban y las funciones que desempeñaban desde el punto de vista del usuario.

\section{Resultados del grupo de discusión}

A raíz del análisis de los grupos de discusión se establecieron cuatro hipótesis para posteriormente corroborarse con la aplicación de una encuesta representativa de la población chilena con acceso a internet. En la primera, que se relaciona con el tipo de contenido que comentan los usuarios, los participantes reconocieron ser más proclives a la hora de comentar en la televisión abierta que en la de pago, o en aquellos contenidos que se transmiten online. Ello, debido a que el consumo lineal tradicional de contenidos de la televisión en abierto facilita comentar los programas televisivos simultáneamente al horario de emisión y así tienen también la posibilidad de intercambiar opiniones con un mayor número de usuarios que en la televisión de pago. Esta conclusión es coherente con estudios previos que han mostrado que una de las principales motivaciones de los usuarios para opinar sobre una temática es pensar que antes de generar contenido, serán correspondidos por terceros. Por ello es posible creer que mientras más posibilidades tengan los usuarios de que sus comentarios sean considerados por sus contactos, mayor debiera ser su motivación para comentar (Courtois; Mechant; De-Marez, 2011).

El participante n. 3, por ejemplo, reconoció que lo fundamental era "ponerse en el lugar de lo que la mayoría de la gente ve", con el fin de obtener un mayor feedback y reconocimiento de lo compartido. Es más, en los grupos de discusión se concluyó que el consumo de la televisión de pago que realizan, lo ven similar a un consumo bajo demanda (on demand), que es más específico y no depende necesariamente de horarios fijos, por lo que consideran que las probabilidades de coincidir con otros espectadores y comentar los contenidos es mucho menor, y por ello se reducirían las posibilidades de interacción. De esta forma, es posible concluir que:

H1. Los espectadores comentan con mayor frecuencia en programación de televisión abierta que en la de pago.

Con respecto a la influencia que los comentarios ejercen en los televidentes, los participantes de los grupos de discusión reconocieron que el leer lo que opinan sus contactos sobre un contenido televisivo genera un interés concreto por ver dicho contenido. Además, estos comentarios tienen muchas veces un efecto en su conducta, ya sea respondiendo a las opiniones de los demás o cambiando lo que ven en televisión para alinearse con lo que dicen. Estas afirmaciones son coherentes con trabajos previos que han mostrado cómo las personas cambian sus conductas televisivas al conversar con terceros sobre el contenido de televisión, ya que al compartir información que se hace visible en las noticias, por ejemplo, ponen más atención al contenido y/o prologan su tiempo de exposición para estar mejor preparados por si tienen que comentar nuevamente (Wonneberger; Schoenbach; Van-Meurs, 2011). De forma similar, la mayoría de los participantes dijo que la lectura de múltiples comentarios sobre un determinado contenido televisivo en las redes sociales influía directamente en su deseo por conocer más sobre la programación a la que se hacía referencia y muchas veces sí cambiaban el canal o encendían la televisión por estos comentarios. Por ello se prevé que:

H2. Los comentarios leídos en redes sociales sobre programación influyen en los hábitos televisivos de la audiencia.

Una de las motivaciones señaladas por la mayoría de participantes para comentar en redes sociales es el entretenimiento. Los jóvenes tienden a buscar información y noticias a través de fuentes de entretenimiento, y a menudo en la forma del humor como aceptación social, relajación o conectividad (Feldman, 2007). Además, el entretenimiento tradicionalmente ha sido como la principal motivación para ver televisión (Rubin, 1983). Con respecto al consumo online, investigaciones recientes han señalado que la percepción de goce o capacidad para generar entretenimiento al compartir vídeos es el principal factor que explica el compartir este tipo de material (Yang; Wang, 2015). Los modelos que han sido elaborados para comprender la viralización en el mun- 
do online explican que si el material genera risa tiene muchas más posibilidades de compartirse por parte del usuario que si no lo hace (Guadagno et al., 2013). Los participantes de los grupos de discusión afirmaron que cuando ven algún tipo de contenido audiovisual disruptivo -algo fuera de lugar que podría parecer gracioso, por ejemplo-, buscan inmediatamente en las redes sociales qué es lo que se dice al respecto y buscan en YouTube el vídeo de ese contenido subido por otro usuario, para poder verlo. De lo anterior se concluye que:

H3. El entretenimiento es el tipo de contenido que mayor cantidad de comentarios genera en redes sociales.

Si bien los participantes coincidieron en muchas de las motivaciones para compartir aspectos de su experiencia televisiva a través de las redes sociales, se reconocieron diferencias en el uso de las redes sociales para ello. Facebook fue reconocida como la red social preferida para conocer, leer y compartir comentarios con usuarios más cercanos. Esta preferencia, según los participantes, se debía al hecho de que consideran que el impacto tanto de sus comentarios como de los demás es mucho mayor en esta red que en Twitter, ya que tienen más contactos cercanos que podrían interactuar con esa información. El participante n. 7 por ejemplo, afirmó que:

"comparto en Facebook porque quiero que me comenten. No opino en Twitter porque siento que hay tantas cosas, que nadie lo va a ver y se va a perder entre todos los tuits, y finalmente nadie los responde".

Varios participantes reconocieron que no utilizaban los hashtags de Twitter por no exponerse ante el resto de usuarios o abrir una discusión pública:

"si uno ocupa el hashtag, uno se expone, se entrega a la discusión pública, y a veces uno no quiere hablar con extraños, no quiere entrar en una discusión pública" (n. 9).

\section{Facebook se utiliza para conocer lo que los contactos cercanos ven, y Twitter para informarse sobre lo que el público opina}

Esta aproximación es coherente por otra parte con la bibliografía sobre el uso de redes sociales, que ha establecido que Facebook favorece el intercambio de mensajes entre contactos más cercanos que en Twitter, lo que facilita la formación de conexiones más íntimas y por ende aumenta también la interacción con el contenido (Han; Lee, 2014). Esto se debe a que los usuarios conocen mejor a sus contactos (muchas veces son cercanos también en el mundo real) al compararse con Twitter, donde los usuarios generalmente la utilizan para informarse sobre ciertos aspectos de interés (Krämer et al., 2015) y/o recibir información directa sobre lo que otros usuarios opinan de un programa o sobre lo que otros están viendo. Es decir, Twitter vendría a ser una red social para conocer temáticas de contingencia o informativas, y por ende se utiliza más para informarse que para interactuar con personas cercanas que podrían responder a comentarios. De esta forma, podría predecirse que:
H4. Facebook es la red más utilizada para leer los comentarios sobre programas de televisión que escriben los contactos más cercanos, y Twitter lo es para conocer lo que las personas piensan sobre programas de televisión.

\section{Método de la encuesta}

Para corroborar las hipótesis planteadas se aplicó una encuesta a una muestra representativa de la población chilena con conexión a internet, cuya composición se basa en la encuesta Casen. El cuestionario online se envió a 20.000 usuarios que son parte del panel representativo de la población online de Chile que maneja TrenDigital, un think tank establecido en la Universidad Católica de Chile. La encuesta fue respondida por 2.705 usuarios, con una tasa de respuesta de $13,53 \%$. La elección de la muestra se basó en tres variables demográficas: sexo (56,8\% femenino), edad (18 a 24 un $48,2 \% ; 25$ a 34 un 24,3\%; 35 a 44 un $12 \%$ y 45 en adelante un $15,5 \%)$ y región geográfica.

\section{El humor y los aspectos informativos son} las áreas temáticas más comentadas

\subsection{Variables}

\section{Tipo de programación}

Se dividió en cuatro tipos: televisión abierta; por cable; on demand y webcasting.

\section{Impacto de los comentarios}

Se preguntó si habían comenzado a ver un programa o cambiado de canal por comentarios que habían leído en redes sociales (tuits, posteos en Facebook, u otro similar), con una escala de 5 posiciones, donde 1 fue nunca y 5 frecuentemente $(M=2,7 ; D S=0,8)$.

\section{Tipo de contenido}

Basándonos en las motivaciones del uso de las redes sociales, como auto-expresión, entretenimiento, información, mantenimiento de relaciones e interacción social (Hunt; Atkin; Krishnan, 2012), se preguntó a los usuarios "qué probabilidad existía de utilizar estas redes sociales para comentar (temas informativos, lúdicos, de humor o interacción entre otros) en un programa de televisión" con una escala de 5 posiciones, en la que 1 es muy poco probable y 5 muy probable.

\section{Comentarios en televisión}

Se preguntó si habían utilizado redes sociales para leer comentarios sobre programación de otros usuarios mientras veían televisión. Se consideró la sumatoria de las respuestas de Facebook $(75,3 \%)$ y Twitter $(36,5 \%)$, las dos redes sociales más utilizadas por los encuestados.

\section{Uso diferenciado de cada red social}

Se preguntó qué red social utilizaba para saber:

- lo que sus amigos estaban viendo en televisión;

- lo que otros opinaban sobre un programa televisivo.

Para ello se consideró sólo aquellos participantes usuarios de Facebook y Twitter mientras ven televisión, es decir, se controló por el uso que se les da a estas redes sociales 
Tabla 1. Regresión múltiple para cambios de conducta TV ( $N=2.447)$

\begin{tabular}{|l|c|}
\hline & Cambio conductas TV \\
\hline Edad & 0,040 \\
\hline Género (1 = mujer) & 0,010 \\
\hline Nivel socioeconómico & 0,045 \\
\hline Exposición pantallas & $0,075^{* *}$ \\
\hline Lectura comentarios & $0,206^{* * *}$ \\
\hline & \\
\hline Constante & 6,480 \\
\hline$R^{2}$ ajustado $(\%)$ & 5,300 \\
\hline
\end{tabular}

Nota: Todas las entradas son coeficientes estandarizados.

$* p \leq 0,05 * * p \leq 0,01 * * * p \leq 0,001$

\section{Variables de control}

Sexo, edad, nivel socioeconómico y tiempo de exposición frente a pantallas.

\section{Resultados de la encuesta}

Para responder la primera hipótesis se preguntó sobre el tipo de programación que los usuarios habían comentado utilizando redes sociales. La programación abierta fue la alternativa que obtuvo el mayor porcentaje, con un $48,1 \%$, al igual que ocurrió en los grupos de discusión. Le siguió la televisión por cable con un $42,8 \%$, luego webcasting (programación por internet) con un $5 \%$, y finalmente la programación bajo demanda (on demand), con un $2,3 \%$ de las preferencias. Al compararse las medias de televisión abierta con la del cable, se concluyó que la abierta es significativamente más alta que por cable $\mathrm{t}(\mathrm{df}=2287)=20,57, \mathrm{p}<0,001)$, corroborando $\mathrm{H} 1$.

Con respecto al impacto de los comentarios leídos en redes sociales sobre los hábitos televisivos de la audiencia, se utilizó una regresión lineal múltiple, donde la variable dependiente fue la frecuencia con que han alterado su conducta por comentarios que leen en redes sociales, y la in- dependiente la frecuencia con que leen comentarios sobre programación de otros usuarios mientras ven televisión. La tabla 1 muestra una relación positiva entre las dos variables, inclusive cuando se controla por sexo, edad, ingresos y cantidad de tiempo frente a una pantalla $(\beta=0,206, p<0,001)$, corroborando $\mathrm{H} 2$.

Para saber si el tipo de contenido que mayor cantidad de comentarios generaba en redes sociales era el humor, como H3 predijo, se preguntó a los usuarios que habían indicado comentar en redes sociales qué probabilidad existía de que las utilizaran en función de una serie de situaciones. El análisis de las respuestas, como muestra la tabla 2, pone a las respuestas "cuando me da risa" ( $M=3,2 ; \mathrm{DS}=1,36)$ y "ante un chascarro (anécdota curiosa)" ( $M=3,04 ; D S=1,39)$ con una probabilidad de ocurrencia alta con respecto a gran parte de las demás posibilidades. Sin embargo, la alternativa de comentar "cuando detecto un error o una información falsa" obtuvo la preferencia más alta $(M=3,45 ; D S=1,38)$, que representa una motivación más informacional, por lo que la hipótesis 3 fue sólo parcialmente demostrada.

\section{Cuantos más comentarios leen los usua- rios en redes sociales mayor es el efecto que tienen en su conducta televisiva}

Para corroborar la hipótesis número 4 y saber si Facebook se utilizaba más para conocer lo que los contactos cercanos ven en televisión y Twitter lo que otros opinan sobre televisión, el análisis de las respuestas mostró que un 51,4\% de los usuarios de Facebook utilizó esta red social para ver lo que sus amigos están viendo en televisión, mientras que sólo un 32,2\% de los usuarios de Twitter lo hizo con el mismo propósito, demostrando que hay un uso diferenciado en este ítem. Para conocer lo que otros opinan sobre un programa televisivo, sólo un $42,7 \%$ de los usuarios de Facebook dijo hacerlo a través de esta red social, mientras que un $70 \%$ de los usuarios de Twitter lo hizo a través de esta red social, corroborando $\mathrm{H} 4$.

Tabla 2. Resultados del análisis descriptivo frecuencia tipo de comentarios ( $N=1.420)$

\begin{tabular}{|c|c|c|}
\hline $\begin{array}{l}\text { ¿Qué probable es que utilice las redes sociales para comentar en los siguientes } \\
\text { momentos de un programa de TV? }\end{array}$ & Media (M) & Desviación estándar (DS) \\
\hline Cuando creo que por el contenido un usuario va a tuitear algo & 2,34 & 1,31 \\
\hline Cuando se dan premios & 2,60 & 1,42 \\
\hline Cuando empiezan los comerciales & 2,56 & 1,36 \\
\hline En todo momento & 1,99 & 1,15 \\
\hline Cuando aparece una escena de sexo & 1,72 & 1,02 \\
\hline Cuando quiero saber el final o las escenas que siguen & 2,01 & 1,27 \\
\hline Cuando me provoca risa & 3,20 & 1,36 \\
\hline Ante un chascarro (anécdota curiosa) & 3,04 & 1,39 \\
\hline Cuando detecto un error o una información falsa & 3,45 & 1,38 \\
\hline
\end{tabular}

Nota: La tabla presenta las medias con escala de 1 a 5 , donde 1 era muy poco probable y 5 muy probable 


\section{Discusión y conclusiones}

La investigación arrojó cinco grandes resultados:

1) Cuantos más comentarios leen los usuarios en redes sociales mayor es el efecto que tienen en su conducta televisiva;

2) La televisión abierta se comenta más que el cable, on demand y webcasting;

3) Las dos redes sociales que mayor cantidad de usuarios tienen -Facebook y Twitter-son también las que más se utilizan para comentar en televisión;

4) El humor y aspectos informacionales son las áreas temáticas que más comentan los usuarios;

5) Hay un uso diferenciado de ambas redes: mientras Facebook es la más utilizada para conocer lo que amigos y cercanos ven en televisión, Twitter lo es para informarse sobre lo que el público general opina sobre el contenido de la televisión.

De los resultados anteriores se desprende una serie de aspectos:

La experiencia de televisión social introduce variables que inciden en el comportamiento de la audiencia y que constituyen un insumo fundamental para la industria

En primer lugar podría establecerse que la participación en televisión a través de redes sociales hoy está enriqueciendo el consumo de contenido audiovisual, ya que para un sector relevante de la población define su experiencia de consumo televisivo, al modificar sus conductas después de leer comentarios. Este aspecto constituye un punto interesante para quienes programan contenido televisivo, pues los comentarios en las redes sociales hacen en ocasiones de llamadas hipertextuales a aquello que se está emitiendo. De este modo, el hecho de que la programación de la televisión abierta sea lo más comentado, da cuenta de una oportunidad tremenda para este sector, que muchas veces se ha visto afectado por el cable. Es decir, para los sectores más conectados, un uso estratégico de las redes sociales podría transformarse en un gancho para atraer a un mayor número de televidentes.

En segundo lugar, es muy interesante que una de las principales motivaciones por parte de los usuarios para comentar en televisión sean los errores, al mostrar una conducta más activa respecto al contenido consumido, ya que además pueden cotejar en la web aquella información emitida que les merezca dudas. Si se mantiene esta corriente parece que las audiencias podrían llegar a tomar incluso un rol más relevante como medidores de calidad de aquello emitido por los medios en el futuro. Así, para el resto de la audiencia y los propios emisores de contenido, la televisión social tendría un valor agregado.
En tercer lugar es relevante el hecho de que los usuarios utilicen Facebook para ver lo que opinan sus amigos, pero que revisen Twitter para averiguar qué es lo que piensan sobre lo que ven. Relacionando este aspecto con el anterior, en los grupos de discusión los usuarios mencionaron que los comentarios sobre un error emitido tiene casi inmediatamente su referencia en Twitter a través de un meme (información viral). Ello le da una utilidad distinta a Twitter frente a Facebook, donde los comentarios sobre televisión se buscan a través de las cuentas de los amigos y más cercanos. Y aunque no se trata de una red social propiamente dicha, YouTube también forma parte de esta experiencia televisiva, ya que los usuarios mencionaron que muchas veces el comentario respecto de un programa de televisión está asociado a un vídeo disponible en YouTube, y es justamente a través de la visualización de este vídeo como también se influye en los hábitos televisivos de la audiencia.

Se observa que esta experiencia de televisión social introduce variables que inciden en el comportamiento de la audiencia y constituyen un insumo fundamental para la industria. Mientras más comentarios leen los usuarios en redes sociales sobre un contenido televisivo, mayor es el efecto que tienen en su conducta televisiva, constituyéndose incluso en un punto de entrada al consumo de televisión. Esto tiene mayores repercusiones para la televisión abierta, ya que es la más comentada, lo que se explica porque hay un mayor número de conocidos en redes sociales que ven este tipo de contenido que en la televisión de pago. Es interesante también observar que a pesar de existir muchas alternativas para participar en redes sociales, cada una de ellas cumple una función específica dada por los propios usuarios cuando ven televisión.

Para un sector relevante de la población, la participación en televisión a través de redes sociales redefine su experiencia de consumo televisivo, al modificar sus conductas después de leer comentarios

\section{Bibliografía}

Arrojo-Baliña, María-José (2013). “La televisión social. Nuevas oportunidades y nuevos retos para el sector audiovisual". En: I Congreso internacional de comunicación y sociedad digital, Logroño.

http://reunir.unir.net/handle/123456789/1729

Cepal (Comisión Económica para América Latina y el Caribe) (2015). La nueva revolución digital: de la internet del consumo a la internet de la producción. http://repositorio.cepal.org/handle/11362/38604

CNTV (Consejo Nacional de Televisión) (2014). VIII encuesta nacional de televisión 2014.

http://www.cntv.cl/prontus_cntv/site/artic/20151209/ asocfile/20151209124713/viii_encuesta_nacional_de_ televisi_n.pdf 
Congosto, Mari-Luz; Deltell-Escolar, Luis; Claes, Florencia; Osteso-López, José-Miguel (2013). “Análisis de la audiencia social por medio de Twitter. Caso de estudio: los premios Goya 2013". Icono 14, v. 11, n. 2, pp. 53-82.

http://dx.doi.org/10.7195/ri14.v11i2.577

Courtois, Cèdric; Mechant, Peter; De-Marez, Lieven (2011). "Teenage uploaders on YouTube: networked public expectancies, online feedback preference, and received on-platform feedback". Cyberpsychology, behavior, and social networking, v. 14, n. 5, pp. 315-322.

http://dx.doi.org/10.1089/cyber.2010.0225

Deltell-Escolar, Luis (2014). “Audiencia social versus audiencia creativa: caso de estudio Twitter". Estudios sobre el mensaje periodístico, v. 20, n. 1, pp. 33-47.

http://dx.doi.org/10.5209/rev_ESMP.2014.v20.n1.45217

Deltell-Escolar, Luis; Claes, Florencia; Osteso-López, JoséMiguel (2013). "Audiencias televisivas y líderes de opinión en Twitter. Caso de estudio: El barco". Estudios sobre el mensaje periodístico, v. 19, pp. 347-364.

http://dx.doi.org/10.5209/rev_ESMP.2013.v19.n1.42526

D'Heer, Evelien; Verdegem, Pieter (2014). "What social media data mean for audience studies: a multidimensional investigation of Twitter use during a current affairs TV programme". Information, communication \& society, v. 18, n. 2, pp. 221-234.

http://dx.doi.org/10.1080/1369118X.2014.952318

Echegaray-Eizaguirre, Lázaro; Peñafiel-Saiz, Carmen (2013). "La utilización de las redes sociales como nuevas herramientas aplicadas al análisis de audiencias". Trípodos, v. 33, pp. 157-172.

http://www.tripodos.com/index.php/Facultat_Comunicacio_ Blanquerna/article/view/102

Fábrega, Jorge; Vega, George (2013). “El impacto del rating televisivo sobre la actividad en Twitter: evidencia para Chile sobre la base del evento Teletón 2012". Cuadernos.info, v. 33, pp. 43-52.

http://www.cuadernos.info/index.php/CDI/article/view/533

Feldman, Lauren (2007). "The news about comedy: young audiences, the daily show, and evolving notions of journalism". Journalism, v. 8, n. 4, pp. 406-427.

http://dx.doi.org/10.1177/1464884907078655

Ferreras-Rodríguez, Eva-María (2014). “Los nuevos prosumidores: audiencias de la televisión social. Análisis de Operación Palace en Twitter". Revista mediterránea de comunicación, v. 5, n. 1, pp. 175-192.

http://dx.doi.org/10.14198/MEDCOM2014.5.2.11

Gallego, Francisco (2013). "Social TV Analytics: nuevas métricas para una nueva forma de ver televisión". Index comunicación, v. 3, pp. 13-39.

http://journals.sfu.ca/indexcomunicacion/index.php/ indexcomunicacion/article/view/49

García-Galera, María-del-Carmen; Valdivia, Angharad (2014). "Prosumidores mediáticos: cultura participativa de las audiencias y responsabilidad de los medios". Comunicar, v. 43, n. XXII, pp. 10-13.

http://dx.doi.org/10.3916/C43-2014-a2
Giglietto, Fabio; Selva, Donatella (2014). "Second screen and participation: a content analysis on a full season dataset of tweets". Journal of communication, n. 64, pp. 260-277. http://dx.doi.org/10.1111/jcom.12085

Gómez, Leire; Vidales, Nereida (2015). “Del éxito en televisión a la participación en las redes sociales. El Príncipe y Galerías Velvet en Facebook". Doxa comunicación, n. 20, pp. 137-160.

http://www.doxacomunicacion.es/es/hemeroteca/ articulos?id $=184$

González-Neira, Ana; Quintas-Froufe, Natalia (2014). “Audiencia tradicional frente a audiencia social: un análisis comparativo en el prime-time televisivo". Revista mediterránea de comunicación, v. 5, n. 1, pp. 105-121. http://dx.doi.org/10.14198/MEDCOM2014.5.1.02

Guadagno, Rosanna; Rempala, Daniel; Murphy, Shannon; Okdie, Bradley (2013). "What makes a video go viral? An analysis of emotional contagion and internet memes". Computers in human behavior, v. 29, n. 6, pp. 2312-2319. http://dx.doi.org/10.1016/j.chb.2013.04.016

Han, Eunyoung; Lee, Sang-Woo (2014). "Motivations for the complementary use of text-based media during linear TV viewing: An exploratory study". Computers in human behavior, v. 32, pp. 235-243. http://dx.doi.org/10.1016/j.chb.2013.12.015

Highfield, Tim; Harrington, Stephen; Bruns, Axel (2013). "Twitter as a technology for audiencing and fandom: The \#Eurovision phenomenon". Information, communication \& society, v. 16, n. 3, pp. 315-339.

http://goo.gl/elkQ5D

http://dx.doi.org/10.1080/1369118X.2012.756053

Hunt, Daniel; Atkin, David; Krishnan, Archana (2012). “The influence of computer mediated communication apprehension on motivations for Facebook use". Journal of broadcasting \& electronic media, v. 56, n. 2, pp. 187-202.

http://macaulay.cuny.edu/eportfolios/sekerina14/ files/2014/09/HON-223-assignment-1a-journal-article.pdf http://dx.doi.org/10.1080/08838151.2012.678717

Krämer, Nicole; Winter, Stephan; Benninghoff, Brenda; GaIlus, Christine (2015). "How 'social' is social TV? The influence of social motives and expected outcomes on the usage of social TV applications". Computers in human behavior, v. 51, pp. 255-262.

http://dx.doi.org/10.1016/j.chb.2015.05.005

Larsson, Anders-Olof (2013). "Tweeting the viewer: use of Twitter in a talk show context". Journal of broadcasting \& electronic media, v. 57, n. 2, pp. 135-152.

https://www.duo.uio.no/bitstream/handle/10852/41499/

jobem_larsson_dec_2012.pdf?sequence $=2$

http://dx.doi.org/10.1080/08838151.2013.787081

Masip, Pere; Guallar, Javier; Suau, Jaume; Ruiz-Caballero, Carlos; Peralta, Miquel (2015). "News and social networks: audience behavior". El profesional de la información, v. 24, n. 4, pp. 363-370.

http://dx.doi.org/10.3145/epi.2015.jul.02

Miranda-Bustamante, María-de-los-Ángeles; Fernández- 
Medina, Francisco (2015). “Hablándole a la televisión: análisis de las conexiones discursivas entre Twitter y tres programas de contenido político". Comunicación y sociedad, v. 24, pp. 71-95.

http://www.redalyc.org/articulo.oa?id=34639625004

Morgan, David (1996). "Focus groups". Annual review of sociology, v. 22, pp. 129-152.

http://goo.gl/c1ovjC

Orozco, Guillermo (coord.) (2013). Tvmorfosis 2. Convergencia y escenarios para una televisión interactiva. México: Tintable. ISBN: 9786079578954

Pynta, Peter; Seixas, Shaun; Nield, Geoffrey; Hier, James; Millward, Emelia; Silberstein, Richard (2014). "The power of social television: can social media build viewer engagement? A new approach to brain imaging of viewer immersion". Journal of advertising research, v. 54, n. 1, pp. 71-80. https://goo.gl/1uTmfm

http://dx.doi.org/10.2501/JAR-54-1-071-080

Quintas-Froufe, Natalia; González-Neira, Ana (2014). “Audiencias activas: participación de la audiencia social en la televisión". Comunicar, v. 43, n. XXII, pp. 83-90.

http://dx.doi.org/10.3916/C43-2014-08

Quintas-Froufe, Natalia; González-Neira, Ana; Díaz-González, María-Jesús (2015). “La construcción de la estrategia comunicativa en Twitter de un falso documental: Operación Palace". Revista latina de comunicación social, v. 70, n. 1, pp. 28-48.

http://dx.doi.org/10.4185/RLCS-2015-1033

Rubin, Alan (1983). "Television uses and gratifications: the interactions of viewing patterns and motivations". Journal of broadcasting \& electronic media, v. 27, n. 1, pp. 37-51.

http://dx.doi.org/10.1080/08838158309386471

Saavedra, Marta; Rodríguez, Leticia; Barón, Gemma (2015). "Audiencia social en España: estrategias de éxito en la televisión nacional". Icono 14, v. 13, pp. 215-237.

http://dx.doi.org/10.7195/ri14.v13i2.822

Segado, Francisco; Grandío, Mar; Fernández-Gómez, Erika (2015). "Social media and television: a bibliographic review on the Web of Science". El profesional de la información, v. 24, n. 3, pp. 227-234.

http://dx.doi.org/10.3145/epi.2015.may.02

Shin, Dong-Hee (2013). "Defining sociability and social presence in social TV". Computers in human behavior, v. 29, n. 3, pp. 939-947.

http://dx.doi.org/10.1016/j.chb.2012.07.006

Webster, James; Ksiazek, Thomas (2012). "The dynamics of audience fragmentation: public attention in an age of digital media". Journal of communication, v. 62, pp. 39-56.

http://dx.doi.org/10.1111/j.1460-2466.2011.01616.x

Wonneberger, Anke; Schoenbach, Klaus; Van-Meurs, Lex (2011). "Interest in news and politics - or situational determinants? Why people watch the news". Journal of broadcasting \& electronic media, v. 55, n. 3, pp. 325-343. http://dx.doi.org/10.1080/08838151.2011.597466

Yang, Chris; Wang, Yingqi (2015). "Social sharing of online videos: examining American consumers' video sharing attitudes, intent, and behavior". Psychology \& marketing, v. 32, n. 9, pp. 907-919.

http://dx.doi.org/10.1002/mar.20826

\section{Inforảrea}

\section{Ayudamos a tu organización en la transformación digital y el gobierno de la información}

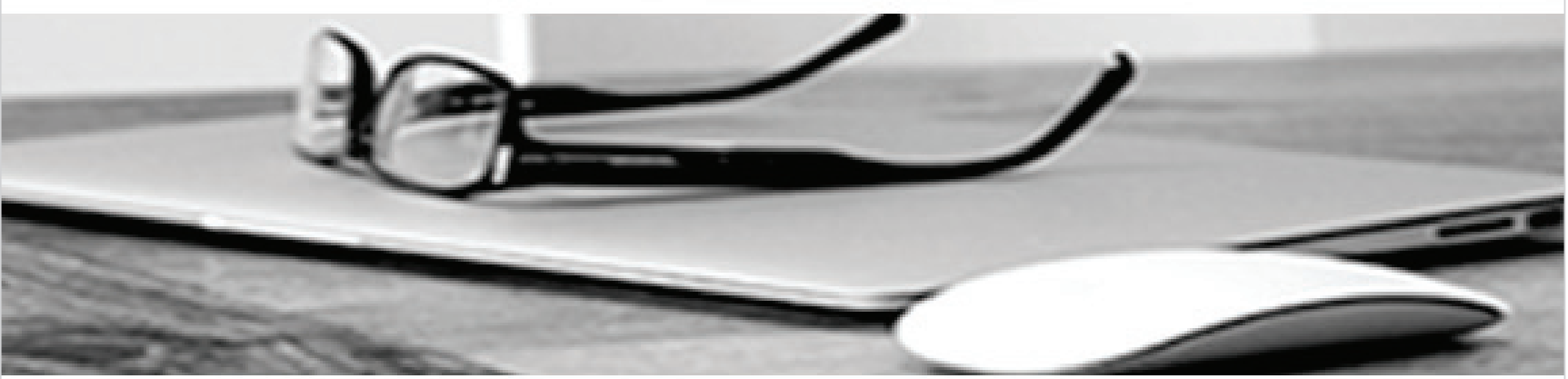

* Consultoría estratégica en gestión y gobierno de la información

" Gestión documental y "records management"

* Gestión de contenidos, intranets corporativas y entornos de colaboración

* Estudios especializados

Clientes satisfechos, cientos de empresas nacionales e internacionales y más de 30 años de experiencia son la mejor garantía de nuestra reputación. 